\title{
Electroweak-QCD interference in hadronic vector bosons decays at the $\mathrm{LHC}$
}

\author{
Cathy Ding ${ }^{1}$, Ben Fuller ${ }^{2}$, Eleanor Jones ${ }^{3}$, Aran Martin ${ }^{4}$, William Murray ${ }^{5, \mathrm{a}}$ (i) \\ ${ }^{1}$ Badminton School, Bristol, UK \\ ${ }^{2}$ Sherborne School, Sherborne, UK \\ ${ }^{3}$ University of Warwick, Coventry, UK \\ 4 The Royal Latin School, Buckingham, UK \\ ${ }^{5}$ STFC Rutherford Appleton Laboratory, University of Warwick, Coventry, UK
}

Received: 23 August 2019 / Accepted: 8 February 2020 / Published online: 26 February 2020

(C) The Author(s) 2020

\begin{abstract}
The analysis of hadronic vector bosons decays at the LHC does not normally consider interference with QCD $q \bar{q}$ production. This paper explores the effect of this interference on the reconstructed peak positions and rates for several production modes. In particular, boosted vectors bosons and vector boson pairs are considered for the first time. Shifts of several $\mathrm{GeV} / \mathrm{c}^{2}$ are seen in the positions of the $W$ and $Z$ bosons' peaks, with a magnitude that depends strongly on the kinematics involved. For boosted vector bosons in regions currently studied, the effects are all found to be very small or negligible. If experiments were to access lower transverse momenta, for example in the experimental trigger systems, or in studies of low-recoil VV decaying semileptonically, the effect of interference could be much larger.
\end{abstract}

\section{Introduction}

This paper presents a study of interference effects between electroweak production of hadronic vector bosons and QCD background at the LHC. Such effects are neglected in the simulation strategy generally adopted by the LHC experimental collaborations, where vector bosons are simulated independently from QCD. The goal of this paper is to test the validity of that approach. Early theoretical studies of interference [1] explored its impact on total $W$ and $Z$ (collectively referred to here as $V$ ) cross-sections in $p p$ and $p \bar{p}$ collisions at energies below $1 \mathrm{TeV} / \mathrm{c}$. More details were explored theoretically at energies of $630 \mathrm{GeV}$ and $1.8 \mathrm{TeV}$ [2] and these studies concluded that "effects caused by the interference between electroweak and QCD amplitudes must be taken into account when data are compared with theoretical predictions". A shift in the masses of the resonance peaks of about $0.3 \mathrm{GeV} / \mathrm{c}^{2}$ was noted and it was also observed that this effect was increased by experimental resolution. First evidence for hadronic vector boson decay was shown in 1990 by UA2 in two-jet decays [3], using $4.66 \mathrm{pb}^{-1}$ of sp⿳亠丷厂s data. This result allowed for a $2.2 \mathrm{GeV} / \mathrm{c}^{2}$ mass shift in the experimental peak position from interference, based on Ref. [2]. Pumplin [4] considered hadronic vector boson observation at the Tevatron as a mass calibration channel and remarked that interference causes a shift in mass of approximately $0.35 \mathrm{GeV} / \mathrm{c}^{2}$ downwards. All of these studies were concerned with inclusive production at leading order in QCD. No experimental evidence for this inclusive process has been published by the LHC experiments.

There are several production modes of boosted hadronic vector bosons at the LHC. Baur [5] considered hadronic $V+$ $X$ production, finding it to be at the order of $1 \%$ of the jet rate. Interest in hadronic vector bosons at the LHC was stimulated by the proposal to analyse boosted hadronic Higgs decays [6] . Inclusive $W \rightarrow q \bar{q}$ decays at high momentum have been used as a calibration in hadronic $X \rightarrow V V \rightarrow q \bar{q} q \bar{q}$ searches $[7,8]$. Both CMS and ATLAS have also used boosted $Z \rightarrow$ $b \bar{b}$ decays as a method of validating techniques to search for highly boosted Higgs bosons decaying to the same final state $[9,10]$. Recently, the ATLAS collaboration performed a cross-section measurement in the $Z(\rightarrow b \bar{b}) \gamma$ channel using $36 \mathrm{fb}^{-1}$ of data [11] and this same channel has also been used to validate $b$-tagging developments [12]. None of these studies considered interference.

Semileptonic VV production provides experimentally accessible signals. For example, the $V H, H \rightarrow b \bar{b}$ discovery papers by ATLAS and CMS $[13,14]$ obtained clear evidence for the $V Z, Z \rightarrow b \bar{b}$ process, summing over $V \rightarrow v v, l v$ and $l^{+} l^{-}$. These searches used lower $p_{\mathrm{T}}$ thresholds on the

a e-mail: bill.murray@ cern.ch (corresponding author) 
bosons than the inclusive ones discussed above, which it will be shown gives rise to larger interference effects.

The multiple distinctive features of top quark pairs allow for the extraction of a clear hadronic $W$ boson signal, which has been used to validate jet performance $[15,16]$ and calibrate the top quark mass measurement $[17,18]$. A shift in the $W$ mass peak here could have a significant impact. This is considered briefly in the concluding section of this paper.

At this stage, it may be helpful to highlight an important point: if two amplitudes $a$ and $b$ contribute to a process, the cross-section is given by $\sigma=a a^{*}+b b^{*}+a b^{*}+b a^{*}$. If $a$ is the process under study and $b$ represents a background, then the fractional contribution of the interference term is at most $2|b| /|a|$. Thus, (fractional) interference effects are expected to scale inversely with the square-root of the signalto-background ratio.

Simulations of several processes are studied at leading order using Sherpa [19] and the procedure is discussed in Sect. 2. At higher order, there are additional effects that are important in the prompt production of heavy quark mesons at hadron colliders. This is especially evident in $\psi(2 s)$ production, where at high $p_{\mathrm{T}}$ even Next-to-Leading-Order QCD (NLO) calculations under-predict the rate by a factor of two [20]. The OpenLoops [21] package was explored as an NLO generator, but no relevant states are currently interfaced to Sherpa. The results for the inclusive vector bosons, for comparison with previous literature, are given in Sect. 3, and, in addition, the analysis methodology is introduced there. This is then applied to inclusive boosted $W$ and $Z$ bosons in Sect. $4, V+\gamma$ in Sect. 5 and $V V$ in Sect. 6. Section 7 contains a discussion of the results and prospects for experimental verification.

\section{Methodology}

The results presented in this paper are obtained using the Sherpa 2.2.8 package with Photos [22] and normally the NNPDF3.0 [23] Parton Distribution Function (PDF) set, with MMHT2014nlo68cl [24] used for certain studies. The Comix [25] matrix element generator is used throughout to generate leading order cross-sections. As such, $s$-channel gluonmediated processes are pure colour octets and do not interfere with vector bosons. Furthermore, the parton shower, hadronization and underlying event are mostly disabled to save computing time, so the resulting output is a small set of partons only. For a small number of examples, the parton shower is enabled and the DelphesMC [26] simulation is employed, using the 'ATLAS' detector card to approximate the influence of a detector on the results. These cases require a jet treatment, discussed in Sect. 3.1.1.
Sherpa uses the concepts of a 'process' and a 'selector'. The process defines the initial and final state particles, with additional options such as defining intermediate states or the order of the electroweak coupling at the amplitude level. The aim of this paper is to study the resonant vector boson peaks, and their interference with the continuum background, but there is no simple way to include all non-resonant electroweak processes in the background. Instead, the three samples generated are a total, a background (which is referred to as QCD throughout), with the minimum electroweak order to reach the specified final state, and the electroweak signal, where two additional orders in the electroweak coupling are required corresponding to the creation and decay of an internal electroweak boson. So, for example, $\gamma u \bar{d}$ is generated at order one and order three in the electromagnetic coupling, though most states involve order zero and order two. The selector imposes kinematic selections on the outgoing particles. In this paper, the selectors applied are:

- a mass window on the relevant quark-antiquark pair of $50 \mathrm{GeV} / \mathrm{c}^{2}<m<130 \mathrm{GeV} / \mathrm{c}^{2}$. This wide window enables studies involving the experimental resolution.

- all final state particles must have a $p_{\mathrm{T}}$ greater than $25 \mathrm{GeV} / \mathrm{c}$. This mimics experimental selection and stabilises the Sherpa integration.

- all charged objects must have $|\eta|<2.5$. This mimics central detector acceptance and also serves to stabilise the Sherpa integration in some cases.

- when studying boosted bosons, the quark-antiquark pair is required to pass a $p_{\mathrm{T}}$ selection. In the case where there are three coloured objects in the final state, this requirement is instead placed on the leading one, which, at this matrix element level, has identical $p_{\mathrm{T}}$ to the recoiling pair.

Simulation is done at $13 \mathrm{TeV}$ centre-of-mass energy for proton-proton collisions. A full 'Run.dat' file defining one process can be seen in appendix A. Within this, it can be seen that the output is in hepMC format. DelphesMC is then used to reformat this into a file that can be processed by root 6.16 [27].

The analysis extracts the interference component as the QCD background and electroweak signal subtracted from the total and comparisons are made between the pure electroweak signal and the electroweak plus the interference term $(E W+I)$. The invariant mass of the $q \bar{q}$ pair is examined. For processes where there are only two quarks in the final state, there is only one combination to form the invariant mass. However, for processes with a $q \bar{q}$ pair plus an extra quark in the final state, there are two possible combinations and both are considered. 


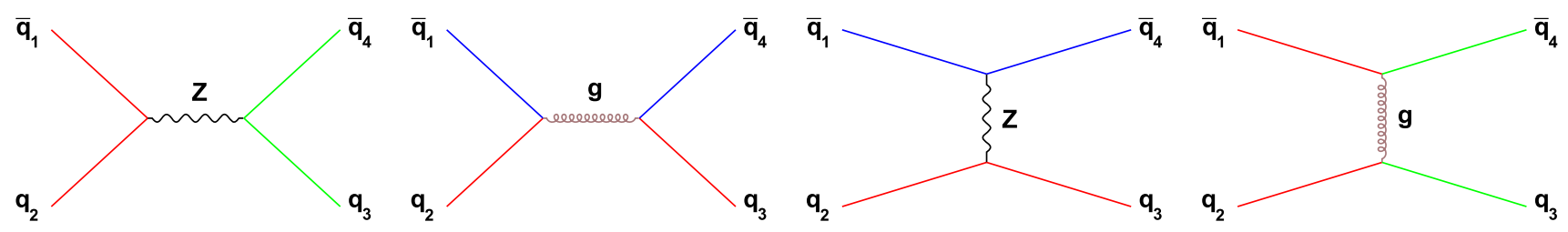

Fig. 1 The leading order $q \bar{q} \rightarrow q \bar{q}$ diagrams, with $s$ - and $t$-channel $Z$ and gluon processes. The $Z$ could be replaced by a $W$ or photon

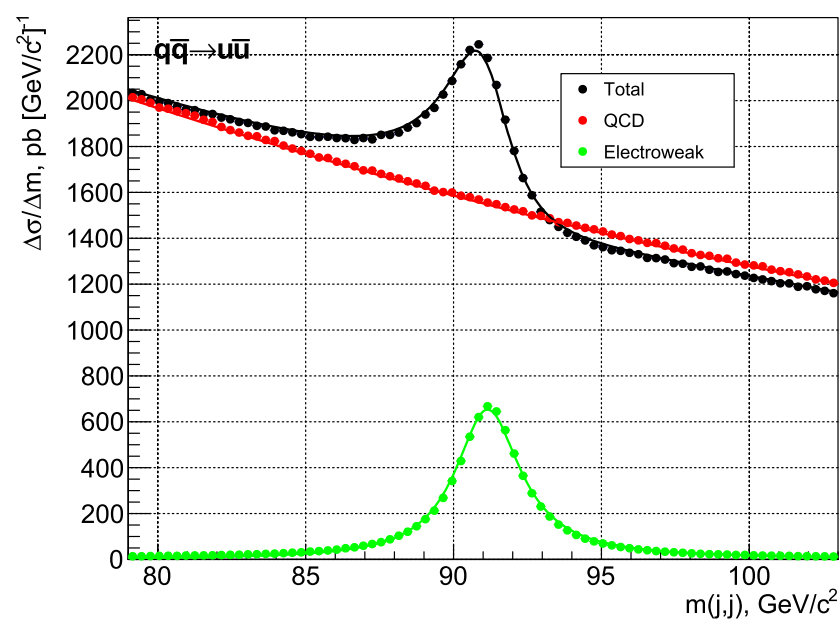

Fig. 2 The process $q \bar{q} \rightarrow u \bar{u}$. Left shows the pure electroweak (pale/green), pure QCD (dark/red) and total (black) cross-sections. Right has the same electroweak component, but the hollow black points

\section{Vector boson production at rest}

In this section, the simplest case of $W$ and $Z$ production with no recoil, $p \bar{p} \rightarrow V$, is considered. This mode shows clear interference effects and so is also used to check the impact of the selection, the experimental resolution and additional gluon radiation.

At leading order, $Z$ or $W$ boson production can only proceed through quark-antiquark annihilation. The experimental background has large contributions from processes involving gluons in the initial or final state. However, interference only occurs between processes with identical initial and final states, so these background processes with gluons will not contribute. To study interference, it is sufficient to focus on $q \bar{q} \rightarrow q \bar{q}$. The total jet-jet cross-section is two orders of magnitude larger than the $u \bar{u}$ cross-section so this represents a considerable calculation simplification. Figure 1 shows the Feynman diagrams for the electroweak and QCD processes.

Only the $s$-channel electroweak process produces a resonant mass signature in the $q \bar{q}$ final state and it is a coloursinglet. However, the similar $s$-channel QCD diagram is a colour octet and, as a result, it does not interfere. This does not hold in general for the $t$-channel gluon exchange process. However, while the experimentalist has some possibility to distinguish final state quarks, all $q \bar{q}$ initial states must be

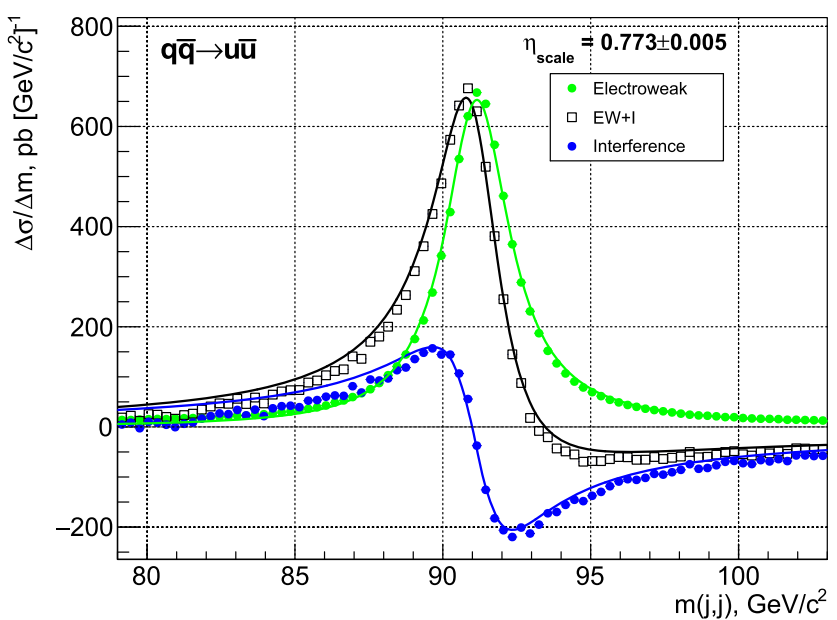

show the total minus the QCD component, and the dark (blue) is the interference term. The fit is discussed in the text

included. This means, for example, that the $t$-channel QCD diagrams for a $b \bar{b}$ final state are suppressed compared to the $u \bar{u}$ final state as a result of the relatively small $b$ quark PDF. Consequently, it is to be expected that the apparent interference is reduced for heavy quarks.

\subsection{Inclusive $Z$ production}

To study $Z$ production, the five final state quark-pairs are simulated separately. The differential cross-section for $q \bar{q} \rightarrow$ $u \bar{u}$ is shown in Fig. 2, with the QCD and electroweak channels compared with the combined process.

There are sizeable interference effects in the mass spectrum, with an enhancement below the peak and destructive interference above. These effects are largest about $\pm \Gamma / 2$ from the pole mass, but they also induce a small shift in the peak position. They are modelled using the sum of a relativistic Breit-Wigner function and an arbitrary constant amplitude:

$\mathcal{A}=\frac{2 m_{0} \sqrt{\kappa\left(m / m_{0}\right)^{3}}}{\left(m^{2}-m_{0}^{2}\right)+i m_{0} \Gamma}+\sqrt{b}+\sqrt{c} i$

In this formula, $\kappa$ is the normalisation of the Breit-Wigner, $m$ is the invariant mass of the system, $m_{0}$ and $\Gamma$ are the particle mass and width, respectively. $b$ and $c$ are the coefficients of 
the real and imaginary amplitude components of an interfering background, respectively. This formula neglects variation in the interfering amplitude with mass. More importantly, in the identification of the $b$ and $c$ terms with the interfering part of the QCD contribution, it ignores $t$-channel electroweak processes. The motivation for the $\mathrm{m}^{3}$ dependence of the Breit-Wigner rate is heuristic.

The cross-section is not just $\mathcal{A A}^{*}$, but has an additional term, $d(m)$, which is a non-interfering background component modelled with a second-order polynomial dependence on $m$ :

$$
\begin{aligned}
\frac{\partial \sigma}{\partial m}= & \frac{2 m_{0} \sqrt{\kappa\left(m / m_{0}\right)^{3}}}{\left(m^{2}-m_{0}^{2}\right)^{2}+m_{0}^{2} \Gamma^{2}} \\
& \times\left(2 m_{0} \sqrt{\kappa\left(m / m_{0}\right)^{3}}+\sqrt{b}\left(m^{2}-m_{0}^{2}\right)+\sqrt{c} m_{0} \Gamma\right) \\
& +b+c+d(m) \mathrm{pb}\left[\mathrm{GeV} / \mathrm{c}^{2}\right]^{-1} .
\end{aligned}
$$

These nine parameters are simultaneously fit to the three distributions in Fig. 2 (left), with terms dropped for the partial distributions where they do not contribute. The extracted values are $m_{Z}=91.125 \pm 0.001 \mathrm{GeV} / \mathrm{c}^{2}\left(\mathrm{c} / \mathrm{f} 91.19 \mathrm{GeV} / \mathrm{c}^{2}\right.$ used in simulation) and $\Gamma_{z}=2.687 \pm 0.003 \mathrm{GeV} / \mathrm{c}^{2}(\mathrm{c} / \mathrm{f}$ $2.50 \mathrm{GeV} / \mathrm{c}^{2}$ used in simulation). The increase in $\Gamma_{z}$ with respect to the simulation is due to the fit attempting to describe the $t$-channel exchange in the electroweak component.

The fit also finds $b=202 \pm 2 \mathrm{pb}\left[\mathrm{GeV} / \mathrm{c}^{2}\right]^{-1}$ and $c=$ $2.2 \pm 0.2 \mathrm{pb}\left[\mathrm{GeV} / \mathrm{c}^{2}\right]^{-1}$; a sizeable real term and a significant imaginary one, which is not expected and is interpreted as being due to the $t$-channel electroweak exchange. The size of the interfering QCD component $b$ is approximately one eighth of the total QCD contribution to $q \bar{q} \rightarrow u \bar{u}$ at $m_{Z}$. The components of the fit are shown in Fig. 2 (right) and it can be seen that the approximate features are captured, but the limitations are clearly visible. The fit is informative, but due to the missing electroweak $t$-channel, it is not quantitatively reliable and simpler methods are generally used for the rest of this paper.

A parameter, $\eta_{\text {scale }}$, characterising the scaling of the signal strength is defined as the integral of the $E W+I$ component in a window of $\pm 10 \mathrm{GeV} / \mathrm{c}^{2}$ around the nominal mass, divided by the same integral for the electroweak term alone. This is the same as the definition of $\eta$ presented by the 'New Physics' Working Group [28], but the integration limits have been reduced from the $\pm 10 \Gamma$ window to one of $\pm 10 \mathrm{GeV} / \mathrm{c}^{2}$, which is comparable to the typical experimental resolution. This revised definition has less sensitivity to the $t$-channel component. The other parameter extracted is the shift of the peak position. This is established by fitting a Gaussian to the observed data, in a mass range defined by the half-maxima of the distribution. The shifts in the peak positions due to interference are evaluated for all the $Z$ decay modes and given in Table 1.

The peak shift, averaged over the final state quark species, is $-0.23 \pm 0.01 \mathrm{GeV} / \mathrm{c}^{2}$, similar to the $0.3-0.35 \mathrm{GeV} / \mathrm{c}^{2}$ shifts reported in the previous work discussed in the introduction, but smaller with the higher beam energy, as expected. The magnitude of the effects falls as the quark mass rises, since the PDF density reduces, and so the non-interfering $s$-channel QCD process becomes more dominant.

These shifts are for the theoretical peak in the crosssection. Estimating the expected change in the measured peak position requires allowing for detector resolution. This was discussed by the 'New Physics' Working Group [29], and Baur and Glover [2], where the latter adopted a $10 \%$ Gaussian smearing to approximate detector resolution effects. The same $10 \%$ is used here, noting that ATLAS found a mass resolution between 8 and $16 \%$ for boosted dijets with $p_{\mathrm{T}}$ between 300 and $1000 \mathrm{GeV} / \mathrm{c}$ [7] and CMS shows between 7.5 and $10 \%$ for $W$ and $Z$ from the decay of $1200-5000 \mathrm{GeV} / \mathrm{c}^{2}$ bosons [8].

Figure 3 (left) shows that after smearing, the $E W+I$ component is negative both above and below the peak, unlike what is seen in the original distribution, as the smearing makes the $t$-channel contribution fractionally larger. The $t$-channel imposes a decrease in differential cross-section across the mass spectrum, and effectively a baseline shift under the peaks. This gives rise to the reported reduction in $\eta_{\text {scale }}$ of 0.69 . If the background had been extracted from a fit to sideband data, this reduction in cross-section would not be seen. The fit to the electroweak alone yields good agreement with the $Z$ mass, while the $E W+I$ distribution is down-shifted by $3.35 \pm 0.14 \mathrm{GeV} / \mathrm{c}^{2}$, as a result of the interference.

This can be compared with the shift in the $q \bar{q} \rightarrow Z \rightarrow u \bar{u}$ peak position before smearing of $-0.405 \pm 0.009 \mathrm{GeV} / \mathrm{c}^{2}$, which shows the experimental resolution increases the shift by an order of magnitude. The larger number can be compared with the systematic error obtained by the experiments on jet energy scale, which in the best measured regions is around $1 \%[30,31]$ or even a little less. It can be seen that the use of vector boson resonances as a standard candle for mass scale is potentially problematic.

The analysis is repeated for only the subprocess $b \bar{b} \rightarrow$ $Z \rightarrow b \bar{b}$ where large effects are expected as the initial and final state quarks are identical. This finds a peak shift of $-0.81 \pm 0.02 \mathrm{GeV} / \mathrm{c}^{2}$, larger than any shift reported in Table 1. This demonstrates that the small interference in the $b \bar{b}$ final state is because its initial state is dominated by the non-interfering lighter-quarks. Figure 3 (right) shows the mass distribution for $b \bar{b} \rightarrow b \bar{b}$ after smearing by $10 \%$, where the change in the peak position is $-6.9 \pm 0.4 \mathrm{GeV} / \mathrm{c}^{2}$. Measuring this distribution would require knowledge of the initial state quarks, which is not experimentally accessible. 
Table 1 Interference effects on the $q \bar{q} \rightarrow W$ peak by final state quark flavour. Signal-to-background is defined at the resonance peak without experimental resolution and using only the initial and final states quoted, with negligible statistical error

\begin{tabular}{llllll}
\hline & $d \bar{d}$ & $u \bar{u}$ & $s \bar{s}$ & $c \bar{c}$ & $b \bar{b}$ \\
\hline Signal-to-background & 0.60 & 0.43 & 1.08 & 1.18 & 2.6 \\
Shift, GeV/c & $-0.384 \pm 0.014$ & $-0.405 \pm 0.009$ & $-0.202 \pm 0.010$ & $-0.135 \pm 0.011$ & $-0.066 \pm 0.007$ \\
$\eta_{\text {scale }}$ & $0.86 \pm 0.01$ & $0.77 \pm 0.01$ & $0.95 \pm 0.01$ & $0.91 \pm 0.01$ & $1.009 \pm 0.003$ \\
\hline
\end{tabular}
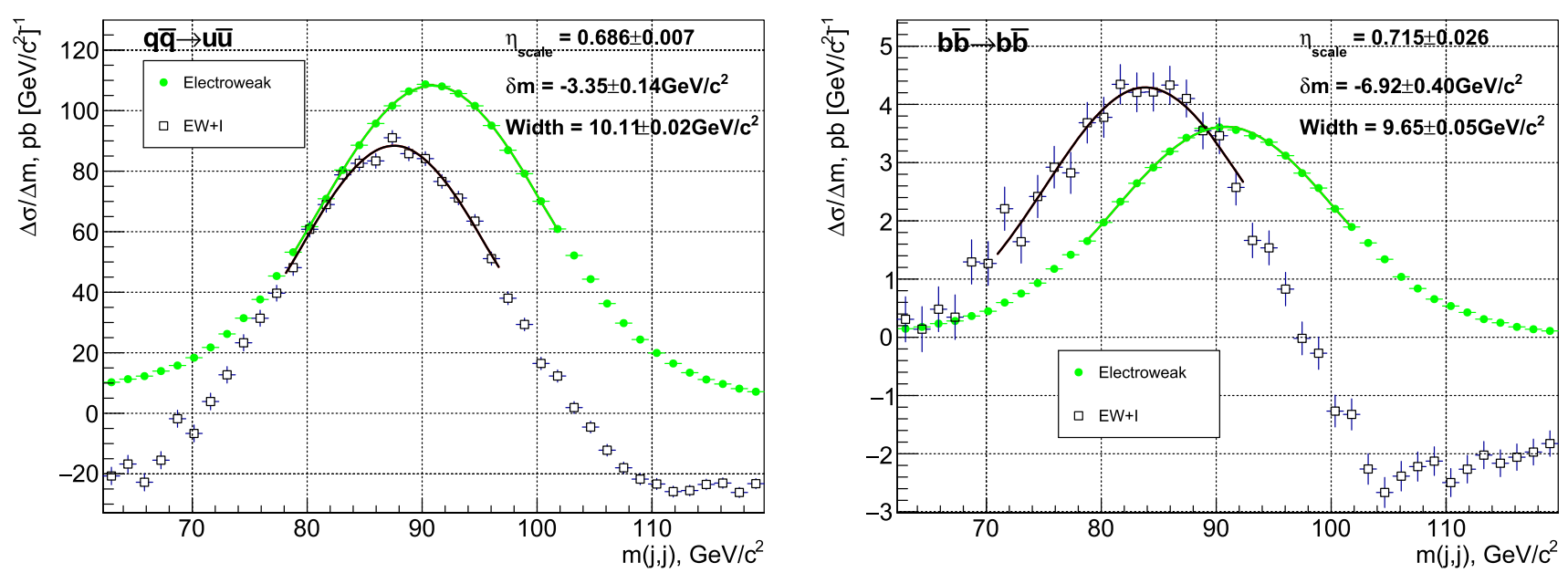

Fig. 3 Left: The process $q \bar{q} \rightarrow u \bar{u}$ after smearing by an assumed $10 \%$ resolution. The electroweak component is pale/green, but the hollow black points show the $E W+I$. Right is the same distribution for $b \bar{b} \rightarrow b \bar{b}$, showing a significantly larger effect

\subsubsection{Variation of procedure}

This section discusses the stability of the results presented above as the physics assumptions involved are varied.

The sensitivity of the peak position to the PDF uncertainties has been explored using the $50 \mathrm{PDF}$ eigenvector variations of MMHT2014nlo68cl. ${ }^{1}$ The quadratic sum of the resulting shifts in the $q \bar{q} \rightarrow Z \rightarrow u \bar{u}$ peak position is $0.006 \mathrm{GeV} / \mathrm{c}^{2}$, corresponding to a $1.5 \%$ uncertainty on the shift. PDF uncertainties are therefore considered negligible.

Variation of the $p_{\mathrm{T}}$ threshold used to select quarks in the process generation has also been studied. This was normally set to $25 \mathrm{GeV} / \mathrm{c}$, but selections of 20 and $30 \mathrm{GeV} / \mathrm{c}$ have been investigated. The change in the pure electroweak crosssection is $\pm 10 \%$, but for the QCD it is a factor of two at $90 \mathrm{GeV} / \mathrm{c}^{2}$. The effect is much larger for QCD because the $t$-channel production tends to have large angular separation. Thus, lower $p_{\mathrm{T}}$ thresholds enlarge the QCD sample in the kinematic region where the colour structure can interfere. The result is that the peak shift has quite a strong dependence on the $p_{\mathrm{T}}$ selection, as seen in Table 2 .

The dependence of the mass shift on the $|\Delta \eta|$ between the quarks is also explored in Table 2. It can be seen that for events where the quarks have similar $\eta$, the $p_{\mathrm{T}}$ threshold has

\footnotetext{
1 Attempting to use the PDF variations of NNPDF30NNLO caused Sherpa termination for memory overflow on a 128 GB RAM computer.
}

little impact, while at large $|\Delta \eta|$, where the $p_{\mathrm{T}}$ can be lower, the threshold is much more important. Thus, the results have a significant dependence on the kinematic selection used.

The shift is also a function of the assumed resolution and a smearing width varying from 0 to $13 \%$ is tested in Fig. 4 . This is compared with the shift extracted from convolving Eq. (2) with a Gaussian of variable width. The match is good, apart from a small offset, until the width reaches $10 \%$, where the impact of the $t$-channel interference, not included in the equation, becomes important.

The figure also shows the result of three progressively more complete ways of introducing resolution: allowing a parton shower, allowing a parton shower and an additional hard parton in the matrix element, and a full hadronization plus the use of Delphes detector simulation, with the ATLAS detector simulation employed. The resulting particles are all analysed using Delphes, with the anti- $\mathrm{k}_{t}$ [32] jet algorithm employed to order the resulting particles. The events are required to have precisely two jets above the $25 \mathrm{GeV} / \mathrm{c}$ $p_{\mathrm{T}}$ threshold and, in the case of the Delphes simulation, the generator threshold is lowered to $15 \mathrm{GeV} / \mathrm{c}$, with the reconstructed jets still required to exceed $25 \mathrm{GeV} / \mathrm{c}$, to allow for threshold effects. The two jets are required to be coplanar to 0.1 radians, which reduces events where a lot of energy is missed from the jet. Finally, in the two cases where Delphes was not used, a $10 \%$ detector smearing is applied to the mass. 
Table 2 The electroweak cross-section accepted within a window of $89-93 \mathrm{GeV} / \mathrm{c}^{2}$ in $m_{u \bar{u}}$, selecting the $Z$ peak, as well as the fitted shift in that peak due to interference. These are given for various quark $p_{\mathrm{T}}$ thresholds and diquark $\Delta \eta$ selections. A $p_{\mathrm{T}}$ threshold of $30 \mathrm{GeV} / \mathrm{c} \mathrm{com}$ bined with a $|\Delta \eta|>2$ essentially excludes the $Z$ boson peak, hence there is no entry for that region

\begin{tabular}{|c|c|c|c|c|c|c|}
\hline \multirow{2}{*}{$\begin{array}{l}\text { Quark } p_{\mathrm{T}} \\
\text { Threshold } \\
(\mathrm{GeV} / \mathrm{c})\end{array}$} & \multicolumn{2}{|l|}{ Inclusive } & \multicolumn{2}{|l|}{$|\Delta \eta|<1$} & \multicolumn{2}{|l|}{$|\Delta \eta|>2$} \\
\hline & Cross-section (pb) & $\begin{array}{l}\text { Peak shift } \\
\left(\mathrm{GeV} / \mathrm{c}^{2}\right)\end{array}$ & $\begin{array}{l}\text { Cross-section } \\
(\mathrm{pb})\end{array}$ & $\begin{array}{l}\text { Peak shift } \\
\left(\mathrm{GeV} / \mathrm{c}^{2}\right)\end{array}$ & $\begin{array}{l}\text { Cross-section } \\
\text { (pb) }\end{array}$ & $\begin{array}{l}\text { Peak shift } \\
\left(\mathrm{GeV} / \mathrm{c}^{2}\right)\end{array}$ \\
\hline 20 & 1882 & $-0.465 \pm 0.017$ & 954 & $-0.280 \pm 0.017$ & 283 & $-0.933 \pm 0.045$ \\
\hline 25 & 1741 & $-0.405 \pm 0.009$ & 945 & $-0.309 \pm 0.008$ & 156 & $-0.737 \pm 0.056$ \\
\hline 30 & 1564 & $-0.351 \pm 0.009$ & 945 & $-0.302 \pm 0.010$ & 0.2 & n.a. \\
\hline
\end{tabular}

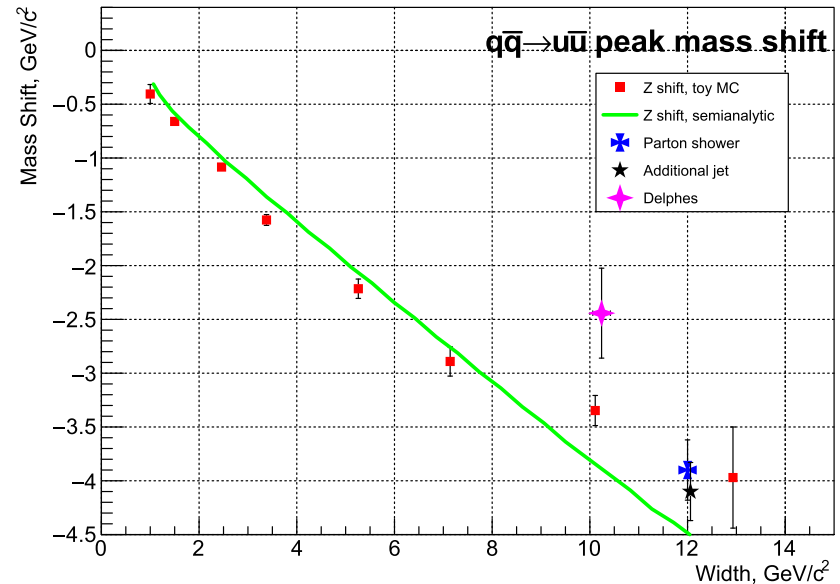

Fig. 4 The shift in the peak positions of Gaussian distributions fitted to $u \bar{u}$ (left)and $b \bar{b}$ (right) peaks, plotted against the fitted width. The squares show the impact of different smearing resolutions, and the star

The resulting peak widths are $12.0 \mathrm{GeV} / \mathrm{c}^{2}$ for the parton shower, $12.1 \mathrm{GeV} / \mathrm{c}^{2}$ with additional hard radiation and $10.2 \pm 0.2 \mathrm{GeV} / \mathrm{c}^{2}$ for the Delphes simulation. This happens to match almost exactly the width resulting from the $10 \%$ smearing applied to the quarks. The shift in the peak position is $3.91 \pm 0.28 \mathrm{GeV} / \mathrm{c}^{2}$ for the parton shower, 4.10 $\pm 0.27 \mathrm{GeV} / \mathrm{c}^{2}$ for the parton shower plus possible hard jet, and $2.42 \pm 0.42 \mathrm{GeV} / \mathrm{c}^{2}$ for the Delphes events. These numbers can be visualised in Fig. 4 (left), which shows a generally consistent pattern, with the Delphes results somewhat higher. This has been traced to reduced acceptance for events from Delphes simulation at large $|\Delta \eta|$, presumably because such events have a $p_{\mathrm{T}}$ close to the acceptance threshold and can easily fall below it as a result. This is the region where the interference effects are larger, see Table $2 .^{2}$

The right hand side of Fig. 4 shows a comparison of no smearing, $10 \%$ smearing and parton shower plus Delphes for the $Z \rightarrow b \bar{b}$ case. A very similar pattern to the $u \bar{u}$ is seen, with the effects scaled down by a factor of six.

\footnotetext{
2 These Delphes studies in particular consumed many thousands of CPU hours and over a hundred terabytes of disk.
}

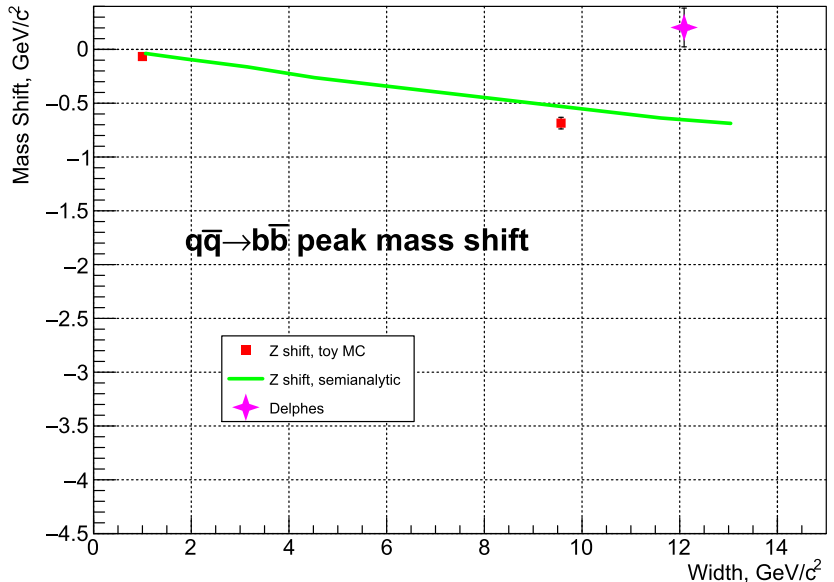

symbols the effect of gluon radiation or Delphes simulation as discussed in the text. The continuous lines show the impact of a numerical smearing applied to the fitted interference formula

\subsection{Inclusive $W$ production}

Inclusive $W$ production is analysed in a manner very similar to the $Z$, but because the $W$ boson is charged it does not have any $s$-channel QCD background. However, this background did not create interference in the $Z$ case and so the actual effects observed are similar. Sherpa is run in its default form, with a diagonal CKM matrix, so the possible signatures are $u \bar{d}, d \bar{u}, c \bar{s}$ and $s \bar{c}$. The peak shifts and scale changes are given in Table 3.

The shifts are found to be similar to, but smaller than, those in the $Z$ case, and the reduction of the effects for secondgeneration quarks is clear, as was also the case for the $Z$ boson.

\section{Boosted vector bosons}

This section describes the dominant boosted production modes, $V q$ and $V g$. These modes have different interference properties, but experimentally, they are almost impossible to distinguish. Recoil against photons or other vector bosons is considered later. 
Table 3 Interference effects on the $q \bar{q} \rightarrow W$ peak by final state quark flavour. Signal-to-background is defined at the resonance peak without experimental resolution and using only the initial and final states quoted, with negligible statistical error

\begin{tabular}{lllll}
\hline & $u \bar{d}$ & $d \bar{u}$ & $c \bar{s}$ & $s \bar{c}$ \\
\hline Signal-to-background & 1.97 & 2.05 & 5.2 & 4.6 \\
Shift, GeV/c & $-0.287 \pm 0.005$ & $-0.242 \pm 0.006$ & $-0096 \pm 0.005$ & $-0.099 \pm 0.005$ \\
$\eta_{\text {scale }}$ & $1.018 \pm 0.003$ & $1.027 \pm 0.004$ & $0.995 \pm 0.002$ & $1.039 \pm 0.003$ \\
\hline
\end{tabular}

Fig. 5 Left: example boosted $W$ boson plus quark production diagram. Centre and Right: example QCD diagrams leading to the same quark state

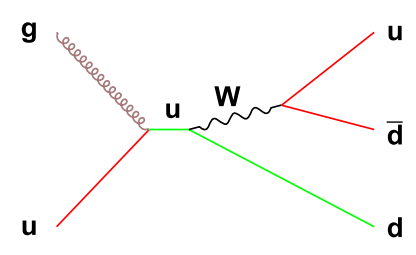

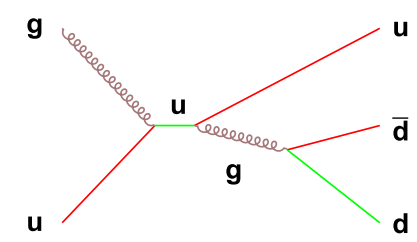

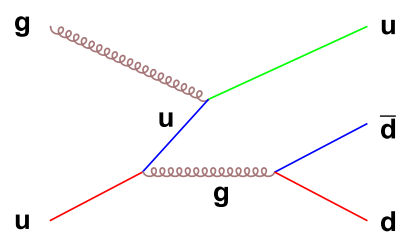

Reference [7] used a $p_{\mathrm{T}}$ selection of $600 \mathrm{GeV} / \mathrm{c}$, but reference [9] clearly shows that an inclusive hadronic $W$ peak could be measured with a $p_{\mathrm{T}}$ selection of $450 \mathrm{GeV} / \mathrm{c}$. ATLAS and CMS used unprescaled jet trigger thresholds of approximately $400 \mathrm{GeV} / \mathrm{c}$ in run 2 at the LHC. In order to imitate the experiments, a $p_{\mathrm{T}}$ threshold of $400 \mathrm{GeV} / \mathrm{c}$ is used as the default here. The extraction of a hadronic $Z$ boson signal experimentally often relies on $b$-quark identification to reduce QCD backgrounds and suppress the larger $W$ peak, so this is given particular attention here.

\subsection{Inclusive boosted $W$}

The processes requested for $W \rightarrow u \bar{d}$ are $q \bar{q} \rightarrow u \bar{d} g$, $q g \rightarrow u \bar{d} q$ or $\bar{q} g \rightarrow u \bar{d} \bar{q}$. It is required that there is a $u \bar{d}$ quark pair with a mass of $50-150 \mathrm{GeV} / \mathrm{c}^{2}$, and as none of the diagrams allow for two identical quarks this is unambiguous. In addition, at least one of the partons must have a $p_{\mathrm{T}}$ above the $400 \mathrm{GeV} / \mathrm{c}$ threshold. In general, this means that the highest $p_{\mathrm{T}}$ parton is recoiling against the $u \bar{d}$ pair.

Sample Feynman diagrams are shown in Fig. 5. It is an amusing feature of the QCD process in the centre that the colour label of the internal $u$ quark is transferred first to the gluon and then to the final $d$ quark. Thus there is a $u$ and $\bar{d}$ pair that is a colour singlet in both the electroweak and this QCD diagram. This does not, however, apply to the right-hand diagram as the incoming gluon changes the colour structure.

The cross-sections of the electroweak process $(W \rightarrow u \bar{d})$ are $0.11 \mathrm{pb}$ for the $W g$ process and $0.23 \mathrm{pb}$ for the $W q$ process in a $2 \mathrm{GeV} / \mathrm{c}^{2}$ window around the $W$ peak. The signal-tobackground ratios at the peak of the resonance are very large, 115 and 681 respectively. This high signal-to-background ratio implies that interference effects must be small. Indeed, this is observed: the values of $\eta_{\text {scale }}$ are $1.004 \pm 0.002$ for the $W g$ process and $1.000 \pm 0.002$ for the $W q$ process, and the corresponding mass shifts are $-0.005 \pm 0.004 \mathrm{GeV} / \mathrm{c}^{2}$ and $0.007 \pm 0.004 \mathrm{GeV} / \mathrm{c}^{2}$ respectively.
For the $W g$ process, the interfering QCD diagrams have the same quark flavours in the initial and final state, much like the inclusive production. Therefore, interference effects will be smaller for $W \rightarrow c \bar{s}$ than for $W \rightarrow u \bar{d}$, in the same way as described for production at rest. Conversely, for the $W q$ process, only one of the final state quarks comes from the initial state and as a result, the suppression will not be as strong. The effects are so small because the $W$-like quark pair in QCD must come from different vertices.

The analysis has been repeated with a threshold of 200 $\mathrm{GeV} / \mathrm{c}$, where the interfering QCD component is less suppressed. The signal-to-background ratios fall by a factor of four and the resulting peak shift for $W g \rightarrow u \bar{d}$ is $0.011 \pm$ $0.005 \mathrm{GeV} / \mathrm{c}^{2}$, and for $W q$ it is $-0.001 \pm 0.004 \mathrm{GeV} / \mathrm{c}^{2}$. Thus, even for a threshold far below that currently employed for an unprescaled jet trigger, interference can be safely neglected.

\subsection{Inclusive boosted $Z$}

The inclusive boosted $Z$ production has many features in common with the $W$, with possible recoil on a gluon, quark or antiquark considered. The $Z \rightarrow b \bar{b}$ channel is studied here and it is also compared with $Z \rightarrow u \bar{u}$, which is expected to show larger interference. The simulation is very similar to the $W$ above, except that the $q \bar{q}$ pair of interest is either $b \bar{b}$ or $u \bar{u}$. Unlike the $W$ case, two identical quarks can be produced, and as the Sherpa selector acts on all matching combinations, it is changed to require a mass of $50 \mathrm{GeV} / \mathrm{c}^{2}$ or more. If this had not been done, the joint requirements that one of the partons has a $p_{\mathrm{T}}$ above $400 \mathrm{GeV} / \mathrm{c}$ and the upper mass cut on both $q \bar{q}$ pairs would together have eliminated like-quark combinations.

Sample Feynman diagrams are shown in Fig. 6. The rightmost diagram creates a colour singlet $b \bar{b}$ final state without requiring a $b$ quark in the initial state; there are other dia- 

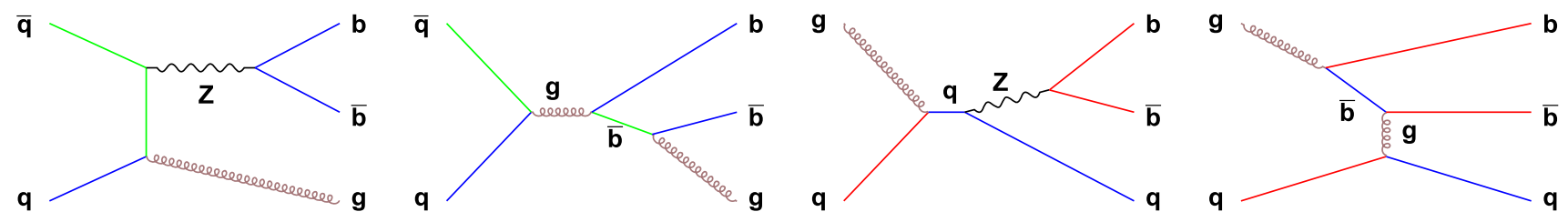

Fig. 6 Feynman diagrams showing the production of a $q \bar{q}$ pair recoiling against either a quark or a gluon. The QCD examples drawn are selected such that they can exhibit interference

Table 4 Interference effects on the $Z$ and $W$ boson peaks with an initial state of either $q g$ plus $\bar{q} g$ or $q \bar{q}$ and separated by final state quark flavour. Signal-to-background is defined at the resonance peak with- out experimental resolution and using only the initial and final states quoted, with negligible statistical error

\begin{tabular}{|c|c|c|c|c|c|c|c|c|c|}
\hline $\begin{array}{l}\text { Recoil } \\
\text { particle }\end{array}$ & $p_{\mathrm{T}}, \mathrm{GeV} / \mathrm{c}$ & $\begin{array}{l}\text { Boson } \\
\text { mode }\end{array}$ & $\begin{array}{l}\text { Signal/ } \\
\text { background }\end{array}$ & $\eta_{\text {scale }}$ & $\begin{array}{l}\text { Peak shift } \\
\left(\mathrm{GeV} / \mathrm{c}^{2}\right)\end{array}$ & $\begin{array}{l}\text { Boson } \\
\text { mode }\end{array}$ & $\begin{array}{l}\text { Signal/ } \\
\text { background }\end{array}$ & $\eta_{\text {scale }}$ & $\begin{array}{l}\text { Peak shift } \\
\left(\mathrm{GeV} / \mathrm{c}^{2}\right)\end{array}$ \\
\hline \multirow[t]{4}{*}{ Gluon } & \multirow[t]{2}{*}{400} & $Z \rightarrow u \bar{u}$ & 9.3 & $1.004 \pm 0.002$ & $+0.004 \pm 0.005$ & \multirow[t]{2}{*}{$W \rightarrow u \bar{d}$} & \multirow[t]{2}{*}{116} & \multirow[t]{2}{*}{$1.000 \pm 0.002$} & \multirow{2}{*}{$-0.005 \pm 0.004$} \\
\hline & & $Z \rightarrow b \bar{b}$ & 21 & $0.996 \pm 0.002$ & $-0.009 \pm 0.005$ & & & & \\
\hline & \multirow[t]{2}{*}{200} & $Z \rightarrow u \bar{u}$ & 3.9 & $1.022 \pm 0.032$ & $+0.022 \pm 0.005$ & \multirow[t]{2}{*}{$W \rightarrow u \bar{d}$} & \multirow[t]{2}{*}{29} & \multirow[t]{2}{*}{$0.996 \pm 0.002$} & \multirow[t]{2}{*}{$+0.011 \pm 0.004$} \\
\hline & & $Z \rightarrow b \bar{b}$ & 21 & $0.996 \pm 0.002$ & $+0.009 \pm 0.004$ & & & & \\
\hline \multirow[t]{3}{*}{ Quark } & \multirow[t]{2}{*}{400} & $Z \rightarrow u \bar{u}$ & 1.05 & $1.016 \pm 0.009$ & $-0.004 \pm 0.007$ & \multirow[t]{2}{*}{$W \rightarrow u \bar{d}$} & \multirow[t]{2}{*}{680} & \multirow[t]{2}{*}{$1.018 \pm 0.002$} & \multirow[t]{2}{*}{$0.007 \pm 0.004$} \\
\hline & & $Z \rightarrow b \bar{b}$ & 1.2 & $1.023 \pm 0.007$ & $-0.002 \pm 0.007$ & & & & \\
\hline & 200 & $Z \rightarrow b \bar{b}$ & 0.91 & $0.958 \pm 0.008$ & $+0.009 \pm 0.004$ & $W \rightarrow u \bar{d}$ & 160 & $1.004 \pm 0.002$ & $-0.001 \pm 0.004$ \\
\hline
\end{tabular}
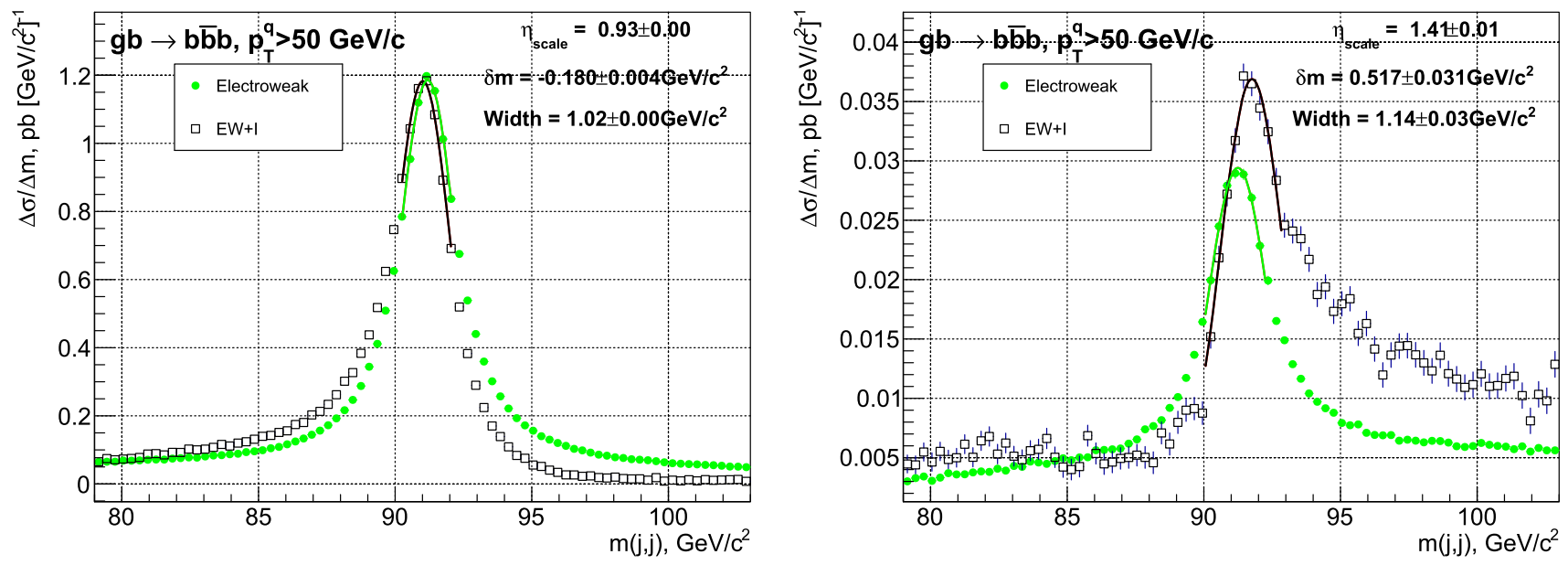

Fig. 7 The process $b g \rightarrow b \bar{b} b$. Left shows events where $|\Delta \eta|<1$ between the $Z$ candidate quarks, while right requires $|\Delta \eta|>2$. Each event generates two $b \bar{b}$ combinations and two entries in these plots if they both fall within the mass range

grams that produce a colour octet $b \bar{b}$ from gluon splitting. These are important differences from the $W$ case.

The signal-to-background ratios and peak shifts are given in Table 4, where results for the $W$ are also included. The signal-to-background ratio is one to two orders of magnitude lower than for boosted $W$ production, so larger shifts might be seen, but most of this comes from gluon splitting. The largest shift is $0.022 \mathrm{GeV} / \mathrm{c}^{2}$, which after applying a $10 \%$ smearing produces a $0.18 \mathrm{GeV} / \mathrm{c}^{2}$ effect. This is negligible compared with the experimental systematic errors.

There is however one more thing which can potentially be measured in the $b \bar{b} q$ state- the flavour of the outgoing quark. If a third $b$ jet is identified, then diagrams like the one in Fig. 6 (right) have enhanced possibilities for interference, as there are two $b \bar{b}$ combinations. Figure 7 shows the electroweak and $E W+I$ contributions when the minimum jet $p_{\mathrm{T}}$ requirement is reduced to $50 \mathrm{GeV} / \mathrm{c}$.

The $b \bar{b}$ pairs with similar pseudorapidity, $|\Delta \eta|<1$, show a significant shift of $-0.17 \mathrm{GeV} / \mathrm{c}^{2}$ at parton level that will correspond to a shift of more than $\mathrm{a} \mathrm{GeV} / \mathrm{c}^{2}$ after detector effects are considered, which is similar to energy scale systematic errors. However, those with a large $|\Delta \eta|$ separation show a dramatic effect, with a shift in the peak position of around $+0.5 \mathrm{GeV} / \mathrm{c}^{2}$. This would produce a shift of several $\mathrm{GeV}$ in a measured spectrum. This effect might be observable at the LHC. For example, the ATLAS collaboration in 
Fig. 8 Example Feynman diagrams for $q \bar{q} \rightarrow q \bar{q} \gamma$. Left is an electroweak process and centre and right are referred to here as QCD
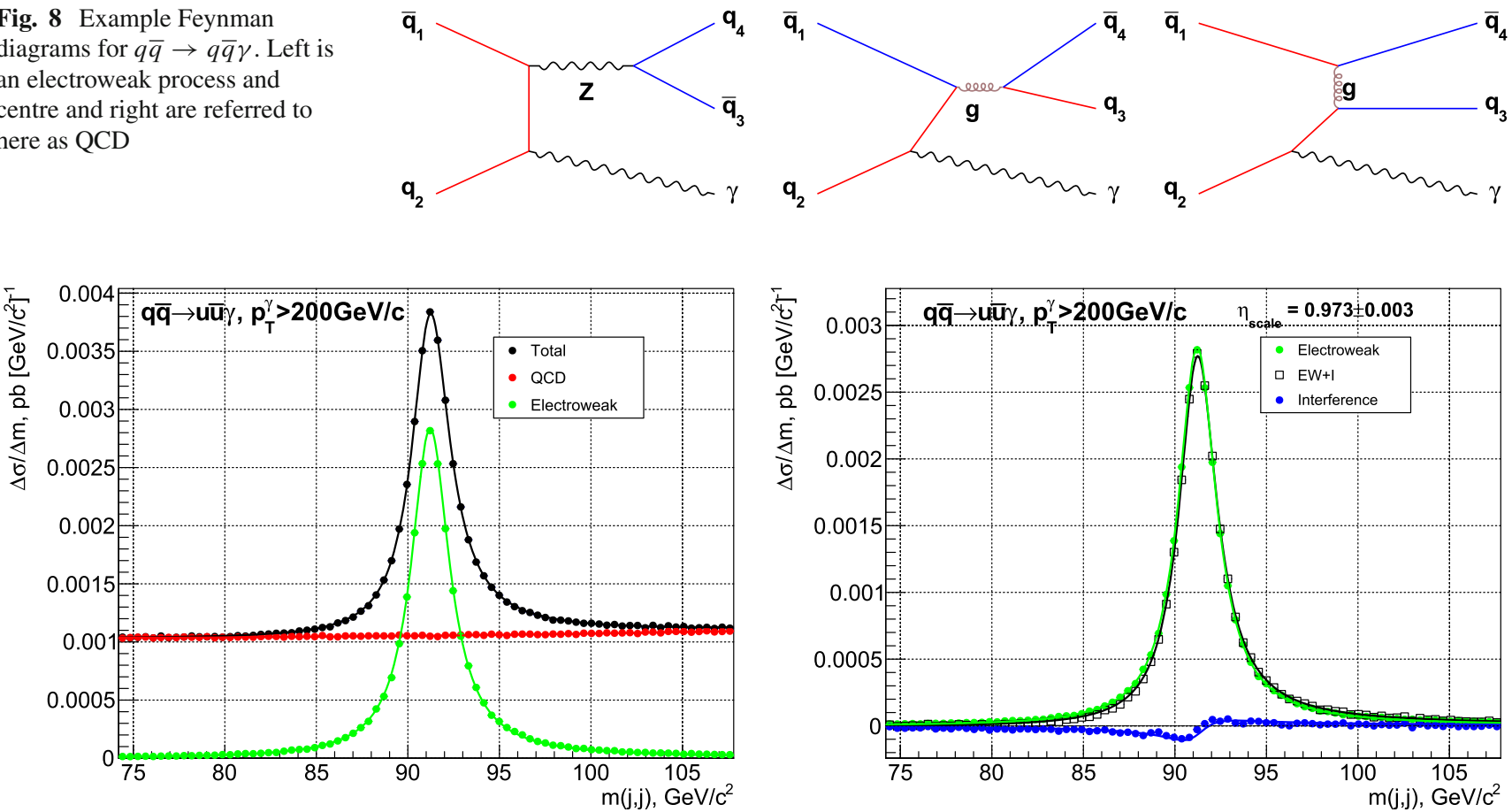

Fig. 9 The cross-section for the process $q \bar{q} \rightarrow u \bar{u} \gamma$, with the $u \bar{u}$ pair required to have $p_{\mathrm{T}}>200 \mathrm{GeV} / \mathrm{c}$. Left shows the pure electroweak (pale/green), pure QCD (dark/red) and total (black) cross-sections.

2016 [33] operated an unprescaled trigger requiring two bjets and a third jet with thresholds of 65,65 and $110 \mathrm{GeV} / \mathrm{c}^{2}$. If an analysis could be performed in the trigger system, or prescaled, it is conceivable that those thresholds could be halved.

\section{$5 V+\gamma$ production}

Studying hadronic vector boson decays recoiling against a photon has significant experimental advantages. Photons couple to charge, so the gluon background is suppressed. ATLAS analysed $\gamma b \bar{b}[11]$, with a trigger on a single photon, an offline $p_{\mathrm{T}}$ selection of $175 \mathrm{GeV} / \mathrm{c}$, and a $Z$ candidate $p_{\mathrm{T}}$ threshold of $200 \mathrm{GeV} / \mathrm{c}$. For the current leading order quarklevel analysis, the $p_{\mathrm{T}}$ of the $V$ and the photon are exactly equal, and $200 \mathrm{GeV} / \mathrm{c}$ is used as a benchmark for these processes. Sample Feynman diagrams are shown in Fig. 8, and results are given in this section for $u \bar{u}, b \bar{b}, u \bar{d}$ and $c \bar{s}$ quark combinations. As usual, the initial state of $g g$, which does not interfere with the electroweak process at leading order, is not included.

As always, the colour structure of $s$-channel gluon production of the quark pair means it does not contribute to interference, although its rate is sizeable. The $t$-channel gluon exchange diagram can interfere, but it preserves the quark species, so while it is important for $u \bar{u} \gamma$, the small PDF suppresses its contribution to the $b \bar{b}$ state.

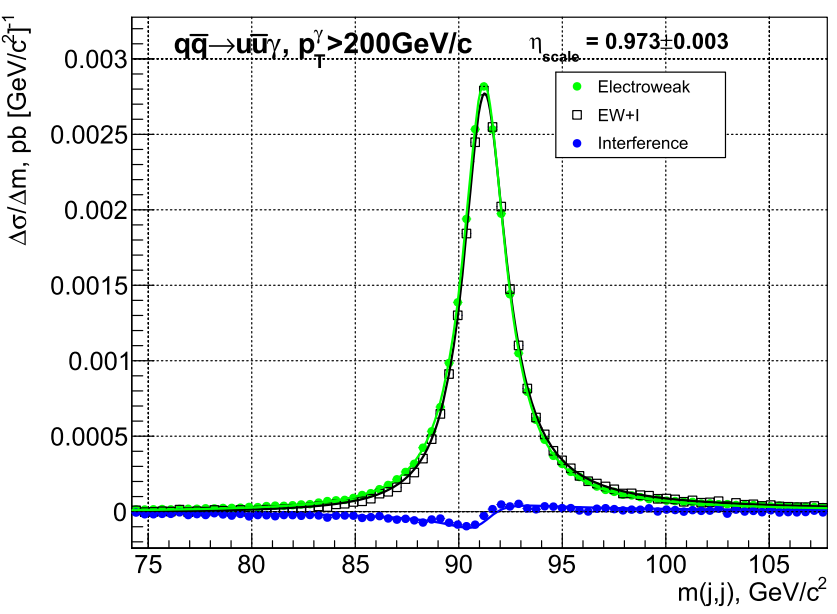

Right has the same electroweak component, but the hollow black points show the total minus the QCD component, and the dark/blue is the interference term

The case of $q \bar{q} \rightarrow Z \rightarrow u \bar{u}$ is shown in Fig. 9. The signal-to-background ratio, on peak, is nearly $3: 1$, but the interfering fraction of the background, fitted as discussed in Sect. 3, is only $0.7 \pm 0.1 \%$. This is consistent with much of the background arising from colour octet gluon splitting. The relative phase of the background is reversed in comparison to the inclusive case, giving a positive shift to the mass peak. The fitted shifts in the peak position are given in Table 5 .

The shift is $0.045 \mathrm{GeV} / \mathrm{c}^{2}$, which, when smeared by $10 \%$, moves the peak position by $0.26 \pm 0.06 \mathrm{GeV} / \mathrm{c}^{2}$. This is significantly below the roughly $1 \%$ experimental systematic errors on the mass scale. The shift in $Z \rightarrow b \bar{b}$ is only $0.008 \mathrm{GeV} / \mathrm{c}^{2}$, far below any observable effects.

A range of $p_{\mathrm{T}}$ selections is explored, from $400 \mathrm{GeV} / \mathrm{c}$ down to $50 \mathrm{GeV} / \mathrm{c}$. The magnitude of the shifts grows as the $p_{\mathrm{T}}$ threshold is reduced. At $50 \mathrm{GeV} / \mathrm{c}$, the $t$-channel electroweak exchange becomes important as evidenced by $\eta_{\text {scale }}$ departing from 1 . Figure 10 shows the differential crosssections for the sub-processes $u \bar{u} \rightarrow u \bar{u} \gamma$ and $d \bar{d} \rightarrow u \bar{u} \gamma$. There is a qualitative difference in the two processes, which highlights the role of the $t$-channel $W$ exchange that is only present in the $d \bar{d} \rightarrow u \bar{u} \gamma$ process; however, both channels show clear interference. With the $50 \mathrm{GeV} / \mathrm{c} p_{\mathrm{T}}$ threshold requirement, the $t$-channel exchange components contribute mostly for masses above the $Z$ mass, and so too does the interference, which suppresses the high-mass cross-section 
Table 5 Interference effects on the $Z$ and $W$ boson peaks in $q \bar{q} \rightarrow V \gamma$, separated by final state quark flavour. Signal-to-background is defined at the resonance peak without experimental resolution and using only the initial and final states quoted, with negligible statistical error

\begin{tabular}{|c|c|c|c|c|c|c|c|c|c|}
\hline $\begin{array}{l}\text { Recoil } \\
\text { particle }\end{array}$ & $p_{\mathrm{T}}(\mathrm{GeV} / \mathrm{c})$ & $\begin{array}{l}\text { Boson } \\
\text { mode }\end{array}$ & $\begin{array}{l}\text { Signal/ } \\
\text { background }\end{array}$ & $\eta_{\text {scale }}$ & $\begin{array}{l}\text { Peak shift } \\
\left(\mathrm{GeV} / \mathrm{c}^{2}\right)\end{array}$ & $\begin{array}{l}\text { Boson } \\
\text { mode }\end{array}$ & $\begin{array}{l}\text { Signal/ } \\
\text { background }\end{array}$ & $\eta_{\text {scale }}$ & $\begin{array}{l}\text { Peak shift } \\
\left(\mathrm{GeV} / \mathrm{c}^{2}\right)\end{array}$ \\
\hline \multirow[t]{8}{*}{ Photon } & \multirow[t]{2}{*}{400} & $Z \rightarrow u \bar{u}$ & 5.2 & $0.999 \pm 0.002$ & $+0.016 \pm 0.005$ & & & & \\
\hline & & $Z \rightarrow b \bar{b}$ & 10.9 & $0.997 \pm 0.002$ & $-0.007 \pm 0.004$ & & & & \\
\hline & \multirow[t]{2}{*}{200} & $Z \rightarrow u \bar{u}$ & 2.7 & $0.973 \pm 0.003$ & $+0.045 \pm 0.005$ & $W \rightarrow u \bar{d}$ & 35 & $1.026 \pm 0.002$ & $+0.007 \pm 0.004$ \\
\hline & & $Z \rightarrow b \bar{b}$ & 9.7 & $1.002 \pm 0.002$ & $-0.008 \pm 0.004$ & $W \rightarrow c \bar{s}$ & 666 & $0.999 \pm 0.002$ & $-0.001 \pm 0.004$ \\
\hline & \multirow[t]{2}{*}{100} & $Z \rightarrow u \bar{u}$ & 1.15 & $0.918 \pm 0.005$ & $+0.065 \pm 0.007$ & & & & \\
\hline & & $Z \rightarrow b \bar{b}$ & 8.1 & $1.010 \pm 0.002$ & $+0.001 \pm 0.004$ & & & & \\
\hline & \multirow[t]{2}{*}{50} & $Z \rightarrow u \bar{u}$ & 0.46 & $0.769 \pm 0.011$ & $-0.073 \pm 0.014$ & $W \rightarrow u \bar{d}$ & 4.3 & $0.953 \pm 0.003$ & $-0.020 \pm 0.005$ \\
\hline & & $Z \rightarrow b \bar{b}$ & 5.5 & $0.987 \pm 0.002$ & $-0.004 \pm 0.004$ & & & & \\
\hline
\end{tabular}

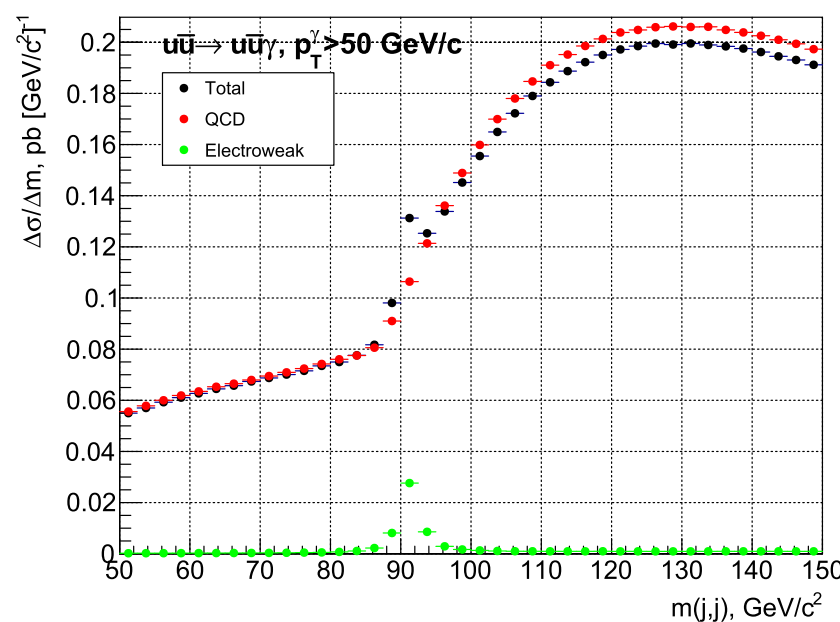

Fig. 10 The differential cross-section for the process $q \bar{q} \rightarrow u \bar{u} \gamma$, with the $u \bar{u}$ pair required to have $p_{\mathrm{T}}>50 \mathrm{GeV} / \mathrm{c}$. Left is $u \bar{u} \rightarrow u \bar{u} \gamma$ and right is $d \bar{d} \rightarrow u \bar{u} \gamma$. The differential cross-section of pure electroweak is pale/green, pure QCD is dark/red and the total is black. The elec-

by approximately $20 \%$. It must be stressed that experimentally these two processes are indistinguishable.

Overall, the interference effects in the $u \bar{u}$ channel are small, but in the experimentally accessed $b \bar{b}$ they are close to zero. Again, the $b$ quark PDF suppresses the interfering background.

The $W+\gamma$ channel is probably experimentally accessible, although there is no published study of the peak. However, as seen in Table 5, the signal-to-background ratio is large, and the $s$-channel interference is vanishingly small. In the same way as for the $Z+\gamma$ channel, at $50 \mathrm{GeV} / \mathrm{c}$, a $t$-channel electroweak component appears and complicates the picture.

\section{Vector boson associated production}

An interesting alternative source of hadronic vector bosons is diboson production, with one of the bosons decaying lep-

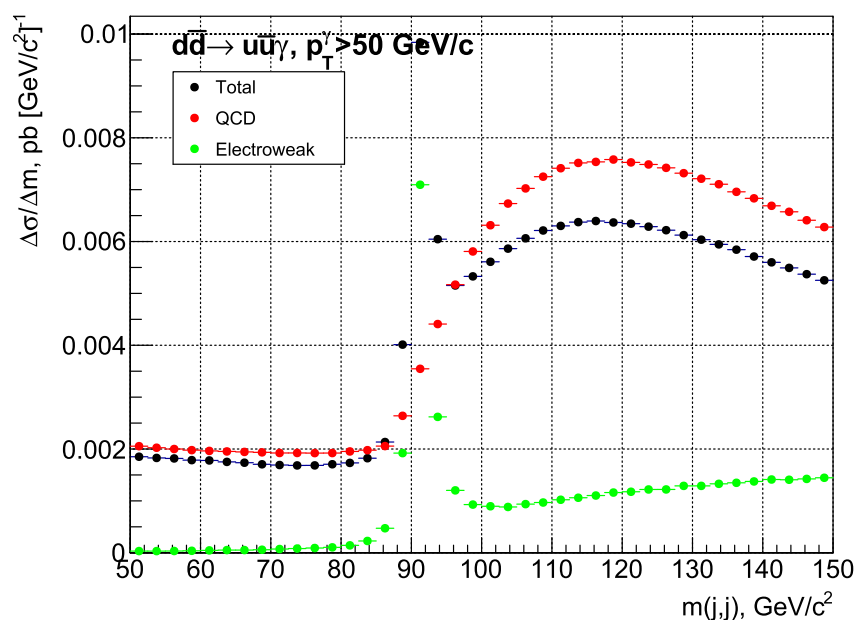

troweak $t$-channel component is much more prominent when $t$-channel $W$ exchange is possible, but in each case the destructive interference is clear

tonically. $W W$ and $Z Z$ decays are explored here, requiring that the leptonic decay involves muons. Such events are triggered efficiently by the LHC detectors, right down to zero recoil. All leptons, charged or neutral, are required to have a $p_{\mathrm{T}}$ above $25 \mathrm{GeV} / \mathrm{c}$ and the angular selections on the muons is $|\eta|<2.5$.

The background is similar to the previously-discussed modes with either $q \bar{q} s$-channel interaction via a colour-octet gluon state, or a $t$-channel diagram in which, for $W W$, the final state $d \bar{u}$ inherits the flavours of the initial $u \bar{u}$ system. The two diagrams are shown on the right-hand side of Fig. 11 and, within the limitations of a diagonal CKM-matrix, only $u \bar{u}$ and $d \bar{d}$ initial states can exhibit the $t$-channel process and thus interfere. The signal has many diagrams, involving one $W^{+}$along with $W^{-}, Z$ or $\gamma$ contributions, but the onshell process sought has either a triple-boson vertex or two separate $W$ boson emissions, as shown in Fig. 11 (left). 

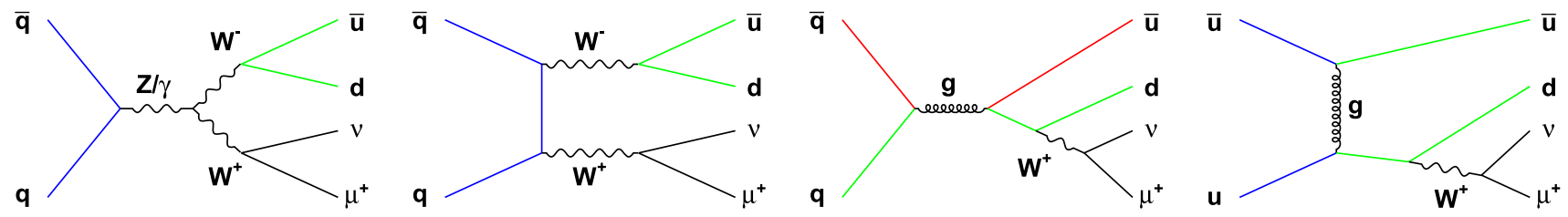

Fig. 11 The leading order $q \bar{q} \rightarrow W^{+} u \bar{d}$ diagrams. The first two diagrams show electroweak processes giving rise to on-shell $W W$ pair production, and the second two are the basic diagrams involving a gluon, referred to here as QCD
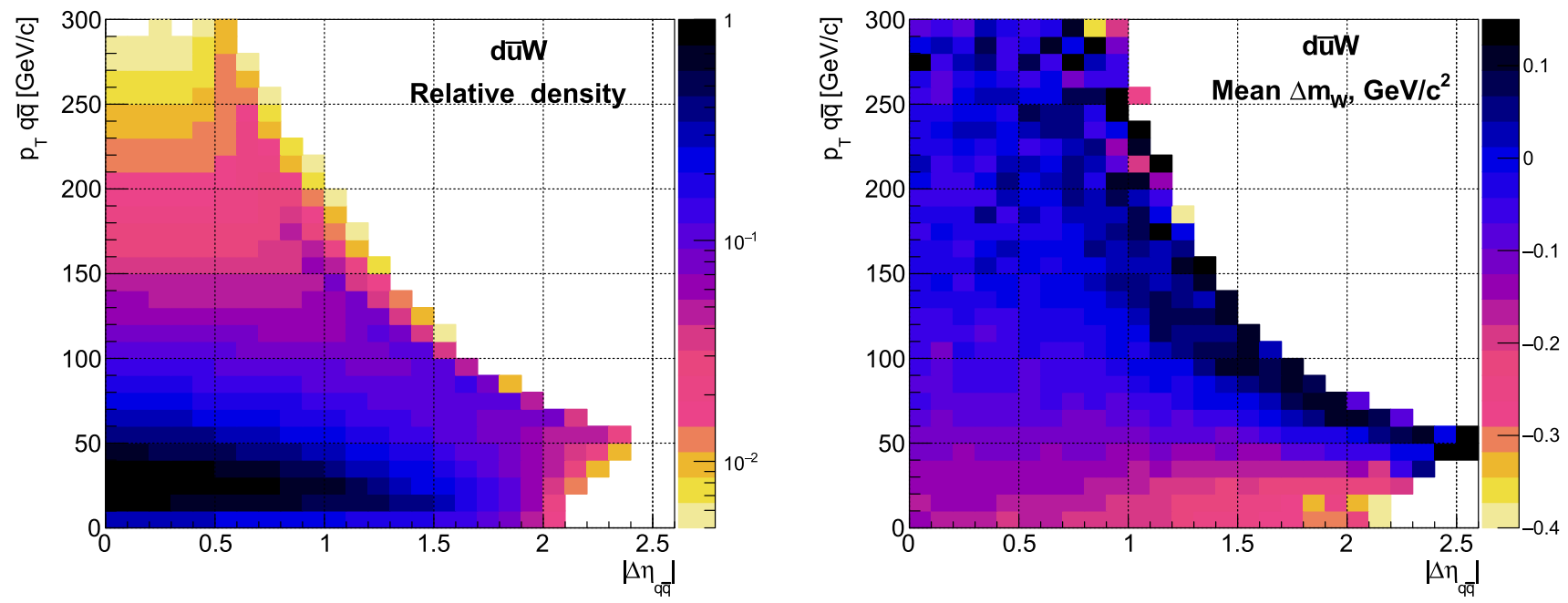

Fig. 12 The process $W W \rightarrow \mu^{+} v d \bar{u}$. Left is the relative density of the electroweak signal as a function of the $|\Delta \eta|$ and $p_{\mathrm{T}}$ of the quark pair. Right is the shift in the mean mass due to interference, as defined in the text

The phase of the two electroweak diagrams shown is opposite, and their relative importance depends upon the kinematics, as does the QCD contribution. The inclusive shift in the peak position in $W^{+} u \bar{d}$ is $0.111 \pm 0.004 \mathrm{GeV} / \mathrm{c}^{2}$, which is significantly smaller than that seen in the prompt production. However, it is dependent on the event kinematics. This is highlighted in Fig. 12, which shows (left) the relative phase space density and (right) the shift in the mean mass. The shift is calculated here by finding the mean mass of $d \bar{u}$ pairs within $\pm 2 \mathrm{GeV} / \mathrm{c}^{2}$ of $80 \mathrm{GeV} / \mathrm{c}^{2}$, and plotting the difference between the electroweak and the difference between total and QCD as follows:

$\Delta m=\frac{\left\langle m_{\text {total }}\right\rangle \sigma_{\text {total }}-\left\langle m_{Q C D}\right\rangle \sigma_{Q C D}}{\sigma_{\text {total }}-\sigma_{Q C D}}-\left\langle m_{E W}\right\rangle$.

This approach, calculating the deviation from the mean in a window, understates any deviation from zero, but the bias has been found to be small.

The most probable kinematics has roughly the shift of $-0.1 \mathrm{GeV} / \mathrm{c}^{2}$ found for the mean, but when there is a pseudorapidity difference of 1.5 or more between the quarks, the shifts range from $-0.25 \mathrm{GeV} / \mathrm{c}^{2}$ at rest to $+0.1 \mathrm{GeV} / \mathrm{c}^{2}$ at recoil momenta approaching $100 \mathrm{GeV} / \mathrm{c}$. Such effects, corresponding to two or three $\mathrm{GeV} / \mathrm{c}^{2}$ after allowing for detector resolution, are potentially observable.
The mean shifts observed in a set of diboson states are summarised in Table 6. $W W$ and $Z Z$ states are explored; $W Z$ are not expected to show qualitatively different behaviour.

The experimental study of such a state would not be able, in general, to distinguish jets from quarks or gluons. The $W^{+} \rightarrow \mu^{+} v j j$ dijet mass spectrum is analysed inclusively for the hadronic $W$ and $Z$ decays, and including all quark or gluon initial state combinations. The $10 \%$ mass resolution is applied, and a mass region from 70.775 to $92.525 \mathrm{GeV} / \mathrm{c}^{2}$ is chosen, defined by the masses where the binned electroweak signal differential cross-section has halved compared with its peak. In that region, the pure electroweak cross-section is $1.3 \mathrm{pb}$, while that for QCD production of the additional jets is $20 \mathrm{pb}$. This corresponds to production of 180,000 events in $140 \mathrm{fb}^{-1}$ of LHC data, or 700,000 if the electron mode and their charge conjugates are also considered, which may be sufficient to allow experimental study.

\section{Discussion}

The impact of electroweak-QCD interference on a variety of hadronic vector boson production modes has been explored. The effect is mostly to move the peak position without changing the integral. There can be destructive interference 
Table 6 Interference effects on the hadronic $Z$ and $W$ boson peaks in $q \bar{q} \rightarrow V V$ with one $V$ decaying muonically and the other separated by final state quark flavour. Signal-to-background is defined at the hadronic resonance peak without experimental resolution and using only the initial and final states quoted, with negligible statistical error

\begin{tabular}{|c|c|c|c|c|c|c|c|c|}
\hline$p_{\mathrm{T}}(\mathrm{GeV} / \mathrm{c})$ & $\begin{array}{l}\text { Boson } \\
\text { mode }\end{array}$ & $\begin{array}{l}\text { Signal/ } \\
\text { background }\end{array}$ & $\eta_{\text {scale }}$ & $\begin{array}{l}\text { Peak shift } \\
\left(\mathrm{GeV} / \mathrm{c}^{2}\right)\end{array}$ & $\begin{array}{l}\text { Boson } \\
\text { mode }\end{array}$ & $\begin{array}{l}\text { Signal/ } \\
\text { background }\end{array}$ & $\eta_{\text {scale }}$ & $\begin{array}{l}\text { Peak shift } \\
\left(\mathrm{GeV} / \mathrm{c}^{2}\right)\end{array}$ \\
\hline \multirow[t]{2}{*}{100} & $Z Z \rightarrow u \bar{u}$ & 6.9 & $0.981 \pm 0.002$ & $-0.005 \pm 0.005$ & \multirow[t]{2}{*}{$W W \rightarrow u \bar{d}$} & \multirow[t]{2}{*}{48} & \multirow[t]{2}{*}{$1.003 \pm 0.001$} & \multirow[t]{2}{*}{$+0.001 \pm 0.003$} \\
\hline & $Z Z \rightarrow b \bar{b}$ & 15.2 & $0.981 \pm 0.002$ & $+0.007 \pm 0.005$ & & & & \\
\hline \multirow[t]{4}{*}{0} & \multirow[t]{2}{*}{$Z Z \rightarrow u \bar{u}$} & \multirow[t]{2}{*}{4.0} & \multirow[t]{2}{*}{$0.885 \pm 0.002$} & \multirow[t]{2}{*}{$-0.117 \pm 0.005$} & $W W \rightarrow u \bar{d}$ & 20.5 & $0.996 \pm 0.002$ & $-0.080 \pm 0.005$ \\
\hline & & & & & $W W \rightarrow d \bar{u}$ & 19.1 & $0.994 \pm 0.002$ & $-0.085 \pm 0.002$ \\
\hline & \multirow[t]{2}{*}{$Z Z \rightarrow b \bar{b}$} & \multirow[t]{2}{*}{13.1} & \multirow[t]{2}{*}{$1.006 \pm 0.002$} & \multirow[t]{2}{*}{$-0.010 \pm 0.004$} & $W W \rightarrow c \bar{s}$ & 86 & $1.011 \pm 0.002$ & $-0.027 \pm 0.004$ \\
\hline & & & & & $W W \rightarrow s \bar{c}$ & 92 & $0.997 \pm 0.002$ & $-0.019 \pm 0.004$ \\
\hline
\end{tabular}

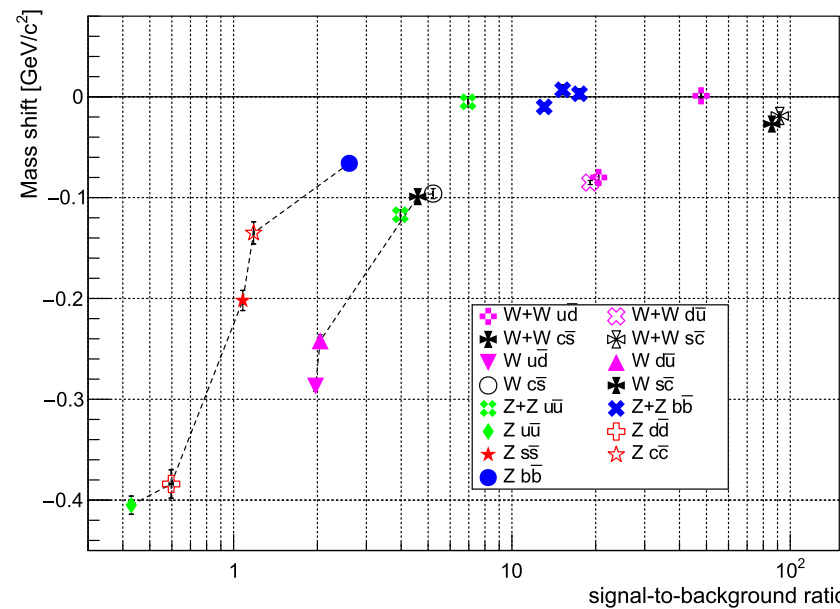

Fig. 13 Summary of the observed shifts in peak positions. Left shows the peak shift as a function of the signal-to-background ratio in the final state, with inclusive production and recoil against a $W$ or $Z$. For both the $W$ and $Z$ produced singly, a dashed line links the different decay

effects between QCD and $t$-channel electroweak diagrams, for example in Fig. 10, but they are not the main focus of this study.

The mass shifts are summarised in Fig. 13, where the lefthand plot shows the production at rest, plotted against the signal-to-background ratio of the quark final state in question. The different decay modes of the $W$ and $Z$ decay at rest are linked to highlight the correlation between large signal-tobackground ratio and small interference [28]. Thus for the $Z$ boson the interference is largest for $u \bar{u}$ decay and smallest for $b \bar{b}$. The $W$ boson does not have a gluon-splitting background, and thus has higher signal-to-background than the $Z$, but the background is more likely to have a colour singlet form, so the interference effects are larger at a given signal-tobackground. The peak shifts fall inside the range spanned by the $Z$ decay modes.

Experiments generally use $b$-tagging to identify $Z$ bosons. This suppresses the $t$-channel QCD processes and reduces the interference effects rather accidentally. If there were a pure and efficient way to identify $u$-quarks available, experiments

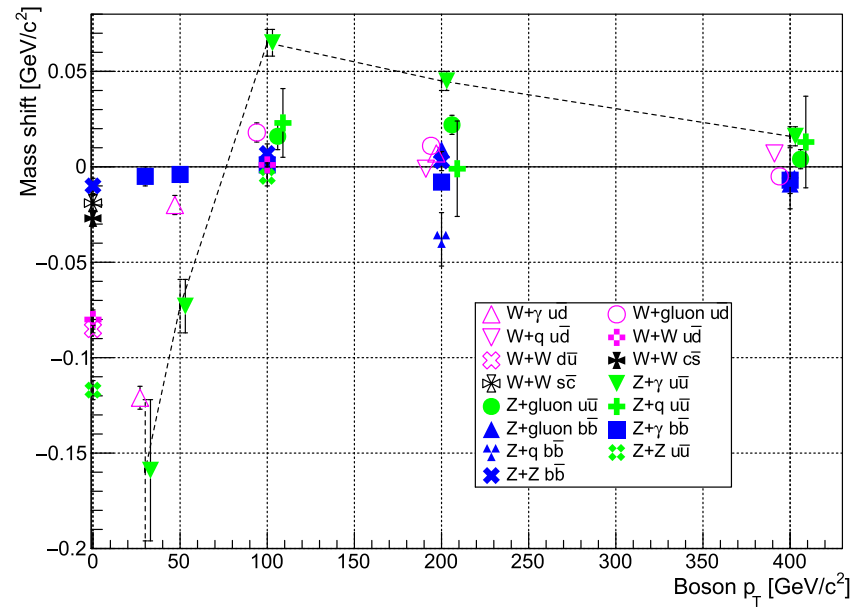

modes. Right shows hadronic vector boson recoiling against $q$, gluon, $\gamma, W$ or $Z$, as a function of the $p_{\mathrm{T}}$ of the vector boson. For the $Z \rightarrow u \bar{u}$, a dashed line links the different $p_{\mathrm{T}}$ selections.

would use that to identify $Z$ candidates too, and would then experience larger interference effects.

It may be possible to study inclusive $Z \rightarrow b \bar{b}$ using the LHC data. The ATLAS and CMS collaborations record data at about $1 \mathrm{kHz}$, from an LHC bunch-crossing rate of around $30 \mathrm{MHz}$. Thus, one event in every 30,000 is recorded as pile-up in the detectors, or approximately $4 \mathrm{pb}^{-1}$. If a $10 \%$ resolution is assumed, the $Z \rightarrow b \bar{b}$ cross-section in a mass window of $80-100 \mathrm{GeV} / \mathrm{c}^{2}$ at leading order is $2500 \mathrm{pb}$, and the QCD background in $b \bar{b}$ is only $8000 \mathrm{pb}$. If non- $b$ background can be suppressed using $b$-tagging, this should be clearly visible.

In the light of the dependence on the signal-to-background ratio in Fig. 13 (left), an explorative simulation of $t \bar{t}$ has been performed. This required the process $g g \rightarrow \mu^{-} \overline{v_{\mu}} \bar{b} d \bar{u} b$, with selections on the two $W$ candidates masses between 50 and $120 \mathrm{GeV} / \mathrm{c}^{2}$, and the top candidate masses between 160 and $190 \mathrm{GeV} / \mathrm{c}^{2}$. The cross-section with two electroweak vertices, one $W$ boson plus QCD jets, is $0.26 \pm 0.01 \mathrm{fb}$, while that at fourth order in electroweak, which includes two on-shell 
top quarks, is $3.01 \pm 0.05 \mathrm{pb}$. This signal-to-background ratio, $10^{4}$, strongly suggests that interference will have a completely negligible impact.

The mass shifts calculated in various boosted states are shown against the vector boson minimum $p_{\mathrm{T}}$ requirement in Fig. 13 (right). As with the bosons at rest, they are largest for $Z \rightarrow u \bar{u}$, and smallest for $Z \rightarrow b \bar{b}$, with the $W$ bosons being intermediate. When vector bosons are boosted to $200 \mathrm{GeV} / \mathrm{c}$ or more, there is generally a positive shift, but it is too small to be experimentally observable. For transverse momenta of $50-100 \mathrm{GeV} / \mathrm{c}$, the patterns are complicated, with the interference swapping sign, partly because the electroweak process is no longer dominated by $s$-channel resonances. The 2D space of $|\Delta \eta|$ between the quarks and the vector boson $p_{\mathrm{T}}$ is presented in Fig. 12 for the $W W$ case, where the varying contributions of TGC production and emission of two independent $W$ bosons, which have opposite phase, leads to opposite sign shifts in different regions.

The shifts in the peak positions are increased by roughly an order of magnitude when smearing the results to mimic resolution effects. For example, an intrinsic shift of $-0.409 \pm$ $0.005 \mathrm{GeV} / \mathrm{c}^{2}$ in $Z \rightarrow u \bar{u}$ is enlarged to $-3.4 \pm$ $0.2 \mathrm{GeV} / \mathrm{c}^{2}$. This is reproduced by an approximate $s$ channel-only interference formula. In this context, the parton shower simulation acts similarly to detector resolution. This is not surprising as it is independently simulated and knows nothing of the interference in the matrix elements. Simulation using Delphes suggests that slightly smaller effects would occur as the jets with large $|\Delta \eta|$ are less likely to be accepted. This could be offset by working with a lower jet $p_{\text {T }}$ threshold than the $25 \mathrm{GeV} / \mathrm{c}$ generally used here.

For the original question that considers whether the boosted hadronic bosons are good standard candles for the calibration of the detectors, the answer is yes. The $W$ decays, especially boosted, have excellent intrinsic signalto-background ratio, while the $Z \rightarrow b \bar{b}$ decay mode is protected by the colour structure of QCD and the small $b$ PDF. The impact of interference on kinematics close to the published measurements has always been found to be small. The one caveat is that NLO effects might give rise to coloursinglet $q \bar{q}$ pairs with a significant cross-section, which would reopen the question for the $Z$ boson. There is no analogous QCD process for $u \bar{d}$ production, so the $W$ boson results should be regarded as more robust. Higgs to $b \bar{b}$ decay has a cross-section significantly lower than $Z$, but its $4 \mathrm{MeV}$ width improves the signal-to-background ratio by 3 orders of magnitude and interference can have no practical impact there.

In future trigger-level analyses, for example in the $W \gamma$ state with a $50 \mathrm{GeV} / \mathrm{c} p_{\mathrm{T}}$ threshold, the $s$ - and $t$-channel interference effects might perhaps be possible to observe. More challenging to record is the $b b \bar{b}$ final state, where large effects should be observable if significant luminosity can be recorded with a $p_{\mathrm{T}}$ threshold of $50 \mathrm{GeV} / \mathrm{c}$. This $Z$ mode might be enhanced by NLO QCD corrections.

However, the $W W \rightarrow l v q \bar{q}$ process seems to have the greatest promise for experimental study. The $W \rightarrow d \bar{u}$ mass peak, after detector resolution, is expected to change position by two or more $\mathrm{GeV} / \mathrm{c}^{2}$ across the kinematic plane. Smaller effects will occur for $c \bar{s}$, but it might be possible to distinguish these final states using charm tagging. When only irreducible background is considered, and pileup neglected, the signalto-background ratio exceeds $5 \%$, so a more detailed study seems warranted. This particular example includes a leptonically decaying boson, so the experiments have recorded this data, and the experimental collaborations are encouraged to see if the predicted effects can be observed.

Acknowledgements Great thanks are due to J. Quevillon who clarified many of these concepts for us and suggested studies and cross-checks, L. Xia, for the helpful observation that at NLO colour octet and singlet merge and to S. Schramm, for the observation that pileup events contribute a significant trigger-free event rate. We also wish to thank our journal referees, whose insistence that we explore the impact of the jet $p_{\mathrm{T}}$ threshold and the use of Delphes simulation highlighted important issues.

Data Availability Statement This manuscript has no associated data or the data will not be deposited. [Authors' comment: The numerical results should be explicitly reevaluated for the precise configuration by anyone concerned. We identify regions where that is necessary. The appendix gives a simple recipe that can be used to do that.]

Open Access This article is licensed under a Creative Commons Attribution 4.0 International License, which permits use, sharing, adaptation, distribution and reproduction in any medium or format, as long as you give appropriate credit to the original author(s) and the source, provide a link to the Creative Commons licence, and indicate if changes were made. The images or other third party material in this article are included in the article's Creative Commons licence, unless indicated otherwise in a credit line to the material. If material is not included in the article's Creative Commons licence and your intended use is not permitted by statutory regulation or exceeds the permitted use, you will need to obtain permission directly from the copyright holder. To view a copy of this licence, visit http://creativecomm ons.org/licenses/by/4.0/.

Funded by SCOAP ${ }^{3}$.

\section{Appendix: Example Run.dat}

An example 'run.dat', for $\mu^{+} v d \bar{u}$ production with a $p_{\mathrm{T}}$ selection set to $0 \mathrm{GeV} / \mathrm{c}$ in the second last line.

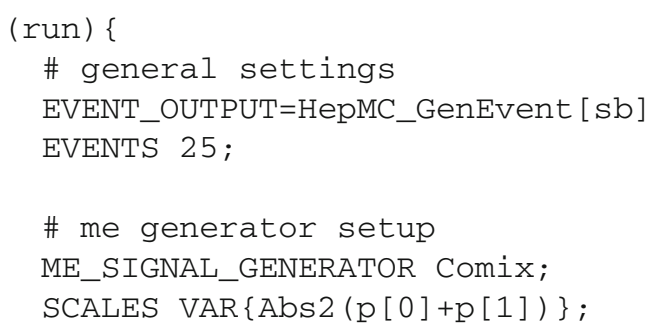


\# Five lines added to switch off decays. SHOWER_GENERATOR=None FRAGMENTATION=Off

MI_HANDLER=None

$M E \_Q E D=O f f$

BEAM_REMNANTS $=0$

\# LHC beam setup:

BEAM_1 2212; BEAM_ENERGY_1 6500;

BEAM_2 2212; BEAM_ENERGY_2 6500;

\} (run)

(processes) \{

Process $94 \quad 94->\quad 1 \quad-2 \quad-13 \quad 14$;

Order $(*, 4)$;

Print_Graphs graphs;

End process;

\} (processes)

(selector) \{

Mass $1-2 \quad 50 \quad 150$

Mass $-13 \quad 14 \quad 70 \quad 90$

PT 9325 E_CMS

PT 9025 E_CMS

PseudoRapidity $93-2.5 \quad 2.5$

"Calc $(\operatorname{PPerp}(\mathrm{p}[0]+\mathrm{p}[1])>0) " 13,-141,1$

\} (selector)

\section{References}

1. G. Ranft, J. Ranft, Phys. Lett. B 87(1-2), 122 (1979). https://doi. org/10.1016/0370-2693(79)90034-0

2. U. Baur, E. Glover, Phys. Lett. B 232(4), 519 (1989)

3. J. Alitti, et al., Z. Phys. C, 49(CERN-PPE-90-105), 17 (1990). https://doi.org/10.1007/BF01570793. https://cds.cern.ch/record/ 210698

4. J. Pumplin, Phys. Rev. D 45, 806 (1992). https://doi.org/10.1103/ PhysRevD.45.806

5. U. Baur, Phys. Rev. D 75, 013005 (2007). https://doi.org/10.1103/ PhysRevD.75.013005

6. J.M. Butterworth, A.R. Davison, M. Rubin, G.P. Salam, Phys. Rev. Lett. 100, 242001 (2008). https://doi.org/10.1103/PhysRevLett. 100.242001

7. The ATLAS collaboration, Aad, G. et al. Search for diboson resonances in hadronic final states in $139 \mathrm{fb}^{-1}$ of $p p$ collisions at $\sqrt{s}=13 \mathrm{TeV}$ with the ATLAS detector (2019). arXiv:1906.08589 [hep-ex]

8. The CMS collaboration, Sirunyan, A. et al. A multi-dimensional search for new heavy resonances decaying to boosted WW, WZ, or ZZ boson pairs in the dijet final state at $13 \mathrm{TeV}$ (2019). arXiv:1906.05977 [hep-ex]

9. The CMS collaboration, Sirunyan, A. et al., Phys. Rev. Lett. 120, 071802 (2018). https://doi.org/10.1103/PhysRevLett.120.071802

10. The ATLAS collaboration, Search for boosted resonances decaying to two b-quarks and produced in association with a jet at $\sqrt{s}=13$ $\mathrm{TeV}$ with the ATLAS detector. Tech. Rep. ATLAS-CONF-2018052, CERN, Geneva (2018). https://cds.cern.ch/record/2649081

11. The ATLAS collaboration, Aad, G. et al. Measurement of the jet mass in high transverse momentum $Z(\rightarrow b \bar{b}) \gamma$ production at $\sqrt{s}=$
$13 \mathrm{TeV}$ using the ATLAS detector (2019). arXiv:1907.07093 [hepex]

12. The ATLAS collaboration, Aad, G. et al., Eur. Phys. J. C 79. arXiv:1906.11005 [hep-ex]. 10, 836. 54 p (2019). https://doi. org/10.1140/epjc/s10052-019-7335-x. https://cds.cern.ch/record/ 2680245. 54 pages in total, author list starting page 38, 20 figures, 3 tables. All figures including auxiliary figures are available at http://atlas.web.cern.ch/Atlas/GROUPS/PHYSICS/ PAPERS/PERF-2017-04

13. M. Aaboud et al., Phys. Lett. B 786, 59 (2018). https://doi.org/10. 1016/j.physletb.2018.09.013

14. The CMS collaboration, Sirunyan, A. et al., Phys. Rev. Lett. 121(12), 121801 (2018). https://doi.org/10.1103/PhysRevLett. 121.121801

15. The CMS collaboration, Jet algorithms performance in $13 \mathrm{TeV}$ data. Tech. Rep. CMS-PAS-JME-16-003, CERN, Geneva (2017). https://cds.cern.ch/record/2256875

16. The ATLAS collaboration, Measurement of large radius jet mass reconstruction performance at $\sqrt{s}=8 \mathrm{TeV}$ using the ATLAS detector. Tech. Rep. ATLAS-CONF-2016-008, CERN, Geneva (2016). https://cds.cern.ch/record/2139642

17. The ATLAS collaboration, Aad, G. et al., Eur. Phys. J. C 79(4), 290 (2019). https://doi.org/10.1140/epjc/s10052-019-6757-9

18. The CMS collaboration, Sirunyan, A. et al., Eur. Phys. J. C 78(11), 891 (2018). https://doi.org/10.1140/epjc/s10052-018-6332-9

19. T. Gleisberg, et al., JHEP 02, 007 (2009). https://doi.org/ 10.1088/1126-6708/2009/02/007. http://www.slac.stanford.edu/ spires/find/hep/www?eprint+0811.4622

20. M. Cacciari, S. Frixione, N. Houdeau, M.L. Mangano, P. Nason, G. Ridolfi, JHEP 10, 137 (2012). https://doi.org/10.1007/ JHEP10(2012)137

21. F. Cascioli, P. Maierhöfer, S. Pozzorini, Phys. Rev. Lett. 108, 111601 (2012). https://doi.org/10.1103/PhysRevLett.108.111601

22. M. Schönherr, F. Krauss, JHEP 12, 018 (2008). https://doi.org/10. 1088/1126-6708/2008/12/018

23. R.D. Ball et al., JHEP 04, 040 (2015). https://doi.org/10.1007/ JHEP04(2015)040

24. L.A. Harland-Lang, A.D. Martin, P. Motylinski, R.S. Thorne, Eur. Phys. J. C 75(5), 204 (2015). https://doi.org/10.1140/epjc/ s10052-015-3397-6

25. T. Gleisberg, S. Höche, JHEP 12, 039 (2008). https://doi.org/10. 1088/1126-6708/2008/12/039

26. The DELPHES 3 collaboration, J. de Favereau, C. Delaere, P. Demin, A. Giammanco, V. Lemaître, A. Mertens, M. Selvaggi, J. High Energy Phys. 2014(2), 57 (2014). https://doi.org/10.1007/ JHEP02(2014)057

27. R. Brun, F. Rademakers, Nucl. Instrum. Meth. A389, 81 (1997). https://doi.org/10.1016/S0168-9002(97)00048-X

28. G. Brooijmans et al., in Les Houches 2017: Physics at TeV Colliders New Physics Working Group Report. Section 19, p145 (2018). http://lss.fnal.gov/archive/2017/conf/ fermilab-conf-17-664-ppd.pdf

29. S.P. Martin, Phys. Rev. D 86, 073016 (2012). https://doi.org/10. 1103/PhysRevD.86.073016

30. M. Aaboud et al., Phys. Rev. D 96(7), 072002 (2017). https://doi. org/10.1103/PhysRevD.96.072002

31. The CMS collaboration, Khachatryan, V. et al., JINST 12(02), P02014 (2017). https://doi.org/10.1088/1748-0221/12/02/P02014

32. M. Cacciari, G.P. Salam, G. Soyez, JHEP 04, 063 (2008). https:// doi.org/10.1088/1126-6708/2008/04/063

33. The ATLAS collaboration, Trigger Menu in 2016. Tech. Rep. ATLDAQ-PUB-2017-001, CERN, Geneva (2017). https://cds.cern.ch/ record/2242069 